\title{
ON THE OPTIMAL CONTROL OF COEFFICIENTS IN ELLIPTIC PROBLEMS. APPLICATION TO THE OPTIMIZATION OF THE HEAD SLIDER
}

\author{
Ionel Ciuperca $^{1}$, Mohamed El Alaoui Talibi ${ }^{2}$ and Mohammed Jai ${ }^{3}$
}

\begin{abstract}
We consider an optimal control problem for a class of non-linear elliptic equations. A result of existence and uniqueness of the state equation is proven under weaker hypotheses than in the literature. We also prove the existence of an optimal control. Applications to some lubrication problems and numerical results are given.
\end{abstract}

Mathematics Subject Classification. 35J25, 49J20, 49J50.

Received July 4, 2002. Revised September 22, 2003 and March 4, 2004.

\section{INTRODUCTION}

In order to achieve increased surface density in a magnetic disk file, the head/disk spacing, which is one of the most critical elements determining the performance of a hard disk drive (HDD), has been continuously reduced. However, the reduction of the head/disk spacing increases the possibility of contact between head slider and disk, leading to a catastrophic failure of the head/disk interface (HDI). To obtain a low and stable flying altitude, it is important to increase the air-bearing stiffness. Finding the gap profile which maximises the load developed by the bearing leads to a higher bearing stiffness and thus to a more stable slider [11]. The slider/disk separation is thin enough for the lubrication hypotheses to hold. This leads to the compressible Reynolds equation [2]:

$$
\begin{cases}\nabla \cdot\left[\left(u^{3} y+6 K u^{2}\right) \nabla y\right]=\Lambda \cdot \nabla(u y) & x \in \Omega \\ y=p_{a} & x \in \partial \Omega\end{cases}
$$

where the air bearing normalized pressure $y=y(x)$ is the unknown of the problem. The normalized film thickness between the head and the magnetic disk is given by $u=u(x)$ which is the control of the problem. $\Omega \subseteq \mathbb{R}^{2}$ is the region (with smooth boundary $\partial \Omega$ ) where the upper (head) and lower (disk) bodies are in proximity. $K>0$ is the so-called Knudsen number, $\Lambda=\left(\Lambda_{1}, \Lambda_{2}\right)$ is the bearing number, and $p_{a}$ is a given function defined on $\bar{\Omega}$.

If we take as a criterion of optimality that of "maximal load" the optimisation problem is

Find $u^{*} \in$ "an appropriate control set" such that $j_{1}\left(u^{*}\right)=\min j_{1}(u)$

\footnotetext{
Keywords and phrases. Compressible Reynolds lubrication equation, optimal control problems, Shauder fixed point theorem.

1 CNRS-UMR 5585 Université Lyon 1, MAPLY, 101, 69622 Villeurbanne Cedex, France.

2 Department of Mathematics, Fac. Sciences Semlalia, 40000 Marrakech, Morocco.

3 CNRS-UMR 5585, INSA de LYON, Mathématiques Bât. Leonard de Vinci, 69621 Villeurbanne, France;

e-mail: mohamed.jai@insa-lyon.fr
} 
with

$$
j_{1}(u)=-\int_{\Omega}\left(y(u)-p_{a}\right) \mathrm{d} x
$$

where $y(u)$ denotes the solution of (1.1) corresponding to an arbitrary control $u$.

This kind of problem also appears in the optimal design of compliant foil journal gas bearing. Using gas as a lubricant allows to increase life durability and to limit the power loss due to shearing of the hydrodynamic film, which makes it attractive for high-speed turbomachinery. In particular, compliant gas bearings supply additional damping and thus increase stability. The counterpart is the lower load capacity with respect to oil lubricated bearings. So it is important to find the optimal shape of compliant gas bearings which maximises the load capacity.

Under the hypothesis $u \in W^{1, \infty}(\Omega)$, Chipot and Luskin [3] have shown the existence and uniqueness of a weak positive solution of (1.1). In [13] Tello proved the existence for the continuum compressible Reynolds equation (Eq. (1.1) with $K=0$ ) in the case where $u$ is a piecewise constant function. He also obtained $W^{1, \infty}$ regularity of the solution in this case. In [4] Diaz and Tello obtained the same result of regularity for the incompressible Reynolds equation. In [7] Grigor'ev et al. proved the existence and uniqueness of a positive solution of the continuum compressible Reynolds equation under hypothesis $u \in C^{1, \gamma}$.

In the first part of this paper we obtain the existence and uniqueness for (1.1) under the weaker assumption $u \in L^{\infty}$.

Very few examples of sliding bearing optimization can be found in the literature. The first and most famous one was investigated by Lord Rayleight [10] who found the one-dimensional step bearing which can carry the largest load for incompressible fluid. Rohde and McAllister [12] numerically computed the best gap profile for a two-dimensional incompressible gas bearing achieving maximum lift force. In the aforementioned examples the gap profile obtained was discontinuous. It is well known that the minimization in Sobolev spaces of type $W^{1, p}(\Omega)$ leads to over-regularization of the control. This brings us to study the problem under weaker hypotheses on $u$ :

- the existence and uniqueness of (1.1) where

$$
u \in L^{\infty}(\Omega), \quad \alpha \leq u(x) \leq \beta \text { a.e. } x \in \Omega \text { with } \alpha, \beta \text { some positive constants; }
$$

- the optimisation problem under the supplementary hypothesis that $u$ belongs to an appropriate closed bounded subset of $B V(\Omega)$.

In fact we consider in this paper a general optimal control problem of the form

$$
\min _{u \in U_{a d}}[j(u)=F(u, y(u))]
$$

subject to

$$
\begin{cases}\nabla \cdot\{[a(x, u(x)) y+b(x, u(x))] \nabla y\}=\nabla \cdot d(x, u(x), y) & x \in \Omega \\ y=g & x \in \partial \Omega \\ y \geq 0 \text { a.e. } x \text { in } \Omega & \end{cases}
$$

where

- $\Omega \subseteq \mathbb{R}^{n}, n \in\{1,2,3\}$, an open bounded subset with smooth boundary $\partial \Omega$;

- $U_{a d}$ is the closed bounded set of the space of the functions of bounded variation in $\Omega$ defined by

$$
U_{a d}=\left\{u \in B V(\Omega) \cap L^{\infty}(\Omega), \alpha \leq u \leq \beta \text { and } T V(u) \leq c\right\}
$$


with $\alpha, \beta, c$ some given positive constants, $B V(\Omega)$ is the Banach space of bounded variation functions and $T V(u)$ is the total variation of $u$ (see $(4.2))$;

- $F: U_{a d} \times H^{1} \longrightarrow \mathbb{R}$;

- $g: \Omega \rightarrow \mathbb{R}$

- $a, b: \Omega \times \mathbb{R} \rightarrow \mathbb{R}^{n^{2}}$

- $d: \Omega \times \mathbb{R} \times \mathbb{R}^{+} \rightarrow \mathbb{R}^{n}$.

In Section 2 we prove the existence and uniqueness of a weak solution $y$ of the following more general problem

$$
\begin{cases}\nabla \cdot\{[\tilde{a}(x) y+\tilde{b}(x)] \nabla y\}=\nabla \cdot \tilde{d}(x, y) & x \in \Omega \\ y=g & x \in \partial \Omega \\ y \geq 0 \text { a.e. } x \text { in } \Omega & \end{cases}
$$

under some weak hypothesis for $\tilde{a}, \tilde{b}: \Omega \rightarrow \mathbb{R}^{n^{2}}, \tilde{d}: \Omega \times \mathbb{R}^{+} \rightarrow \mathbb{R}^{n}$ and $g$.

This will imply the existence and uniqueness of a weak positive solution $y(u)$ of $(\mathrm{P})$ for any $u \in U_{a d}$.

In Section 3 we shall consider the linear elliptic problem

$$
\left\{\begin{array}{l}
-\nabla \cdot(A(x) \nabla y)+\nabla \cdot(B(x) y)=f \\
y \in H_{0}^{1}(\Omega)
\end{array}\right.
$$

with $A: \Omega \rightarrow \mathbb{R}^{n^{2}}, B: \Omega \rightarrow \mathbb{R}^{n}$. We prove existence and uniqueness for $A$ bounded and uniformly elliptic and $B \in L^{r}(\Omega)$ with $r>n$. This hypotheses are weaker than in the literature [6]. The same kind of results will be given for a linear elliptic problem in non-conservative form (the term $\nabla \cdot(B(x) y)$ is replaced by $B(x) \cdot \nabla y)$.

In Section 4, we first prove the existence of a solution of the optimal control problem $(\mathrm{O})$. We then give optimality conditions in the two dimensional case only.

In Section 5 we give some applications to lubrication problems and finally, in the last section we give some numerical results.

\section{Study of the State EQUation}

\subsection{Existence and uniqueness of (PGEN)}

We first study the general nonlinear elliptic problem (PGEN). The hypotheses on data are

$$
\left\{\begin{array}{l}
\tilde{a}=\left(\tilde{a}_{i j}\right)_{1 \leq i, j \leq n} \quad \text { with } \quad \tilde{a}_{i j} \in L^{\infty}(\Omega) \\
\sum_{i, j} \tilde{a}_{i j}(x) \xi_{i} \xi_{j} \geq \gamma\|\xi\|^{2} \quad \text { a.e. } x \in \Omega, \quad \forall \xi \in \mathbb{R}^{n} \quad \text { for a given } \gamma>0 .
\end{array}\right.
$$

We set

$$
\begin{gathered}
\bar{a}=\sup _{i, j}\left\|\tilde{a}_{i j}\right\|_{L^{\infty}(\Omega)} \\
\left\{\begin{array}{l}
\tilde{b}=\left(\tilde{b}_{i j}\right)_{1 \leq i, j \leq n} \quad \text { with } \quad \tilde{b}_{i j} \in L^{\infty}(\Omega) \\
\sum_{i, j} \tilde{b}_{i j}(x) \xi_{i} \xi_{j} \geq \gamma\|\xi\|^{2} \quad \text { a.e. } x \in \Omega, \quad \forall \xi \in \mathbb{R}^{n} .
\end{array}\right.
\end{gathered}
$$


We set

$$
\begin{gathered}
\qquad \bar{b}=\sup _{i, j}\left\|\tilde{b}_{i j}\right\|_{L^{\infty}(\Omega)} \\
\left\{\begin{array}{l}
\tilde{d}_{=}=\left(\tilde{d}_{i}\right)_{1 \leq i \leq n} \quad \text { with } \tilde{d}_{i}(\cdot, y) \text { measurable } \forall y \geq 0 \\
\tilde{d}_{i}(x, 0)=0 \text { a.e. } x \in \Omega \\
\left|\tilde{d}_{i}\left(x, y_{2}\right)-\tilde{d}_{i}\left(x, y_{1}\right)\right| \leq \bar{d}\left|y_{2}-y_{1}\right| \text { a.e. } x \in \Omega \forall y_{1}, y_{2} \geq 0 \quad \text { for some } \bar{d}>0 \\
g \in H^{1}(\Omega), \quad 0 \leq g \leq \bar{g} \text { a.e. } x \in \Omega \quad \text { for some } \bar{g} \geq 0 .
\end{array}\right.
\end{gathered}
$$

In the following, we consider only positive weak solutions of (PGEN) in the sense:

Definition 2.1. A function $y$ is called a positive weak solution of (PGEN) if $y \in g+H_{0}^{1}(\Omega) \cap L^{\infty}(\Omega), y(x) \geq$ 0 a.e. $x$ in $\Omega$ and

$$
\int_{\Omega}(\tilde{a}(x) y(x)+\tilde{b}(x)) \nabla y \cdot \nabla v \mathrm{~d} x=\int_{\Omega} \tilde{d}(x, y(x)) \cdot \nabla v \mathrm{~d} x \quad \forall v \in H_{0}^{1}(\Omega) .
$$

(PVGEN)

\subsubsection{Existence}

To show that (PVGEN) has a solution by using a fixed point procedure, we set for any positive number $R$

$$
B_{R}=\left\{y \in L^{2}(\Omega): 0 \leq y(x) \leq R, \text { a.e. } x \in \Omega\right\}
$$

which is a closed set of $L^{2}(\Omega)$ and define the operator

$$
T: B_{R} \rightarrow H^{1}(\Omega)
$$

by $T y=z$ if $z \in g+H_{0}^{1}(\Omega)$ is the unique solution of the variational problem:

$$
\int_{\Omega}(\tilde{a}(x) y(x)+\tilde{b}(x)) \nabla z \cdot \nabla v \mathrm{~d} x=\int_{\Omega} \tilde{d}(x, y(x)) \cdot \nabla v \mathrm{~d} x \quad \forall v \in H_{0}^{1}(\Omega) .
$$

We introduce also the operator $S$ from $B_{R}$ to $L^{2}(\Omega)$ defined by $S y=(T y)^{+}=\max (T y, 0)$.

From the hypotheses $\left(\tilde{H}_{a}\right)\left(\tilde{H}_{b}\right)\left(\tilde{H}_{d}\right)\left(H_{g}\right)$, it is clear that the problem $(2.3)$ has a unique solution. In order to prove that $S$ has a fixed point by the Shauder fixed point theorem we shall state some preliminary lemmas.

Lemma 2.2. Let $z=T y$ with $y$ arbitrary in $B_{R}$. We have:

$$
\begin{array}{r}
\|\nabla z\|_{L^{2}(\Omega)} \leq C \sqrt{R} \\
z(x) \leq \bar{g}+D \sqrt{R} \quad \text { a.e. } x \text { in } \Omega
\end{array}
$$

where

$$
C \text { and } D \text { depend only on }(\bar{a}, \bar{b}, \gamma, \bar{d}, g, \Omega) .
$$

Proof. Taking $v=z-g$ in (2.3) we obtain

$$
\int_{\Omega}(\tilde{a}(x) y+\tilde{b}(x)) \nabla z \cdot \nabla z \mathrm{~d} x=\int_{\Omega}(\tilde{a}(x) y+\tilde{b}(x)) \nabla z \nabla g \mathrm{~d} x+\int_{\Omega} \tilde{d}(x, y) \cdot \nabla z \mathrm{~d} x-\int_{\Omega} \tilde{d}(x, y) \cdot \nabla g \mathrm{~d} x .
$$

Using $\left(\tilde{H}_{a}\right)\left(\tilde{H}_{b}\right)\left(\tilde{H}_{d}\right)$ and $\left(H_{g}\right)$ and since $y \in B_{R}$ we have

$$
\gamma \int_{\Omega}(y+1)|\nabla z|^{2} \mathrm{~d} x \leq \max (\bar{a}, \bar{b}) \int_{\Omega}(y+1)|\nabla z||\nabla g| \mathrm{d} x+\bar{d} \int_{\Omega}(y+1)|\nabla z| \mathrm{d} x+\bar{d} R \int_{\Omega}|\nabla g| \mathrm{d} x .
$$


Now using $(1+y) \leq \sqrt{2} \sqrt{R} \sqrt{1+y}$ for $R \geq 1$ with the Cauchy-Schwarz inequality we obtain

$$
\int_{\Omega}(y+1)|\nabla z|^{2} \mathrm{~d} x \leq C_{1} \sqrt{R}\left(\int_{\Omega}(y+1)|\nabla z|^{2} \mathrm{~d} x\right)^{\frac{1}{2}}+C_{2} R
$$

where

$$
C_{1}=\sqrt{2} \frac{\max (\bar{a}, \bar{b})\|\nabla g\|_{L^{2}(\Omega)}+\bar{d}|\Omega|^{\frac{1}{2}}}{\gamma} ; \quad C_{2}=\frac{\bar{d}\|\nabla g\|_{L^{2}(\Omega)}|\Omega|^{\frac{1}{2}}}{\gamma}
$$

which implies that there exists a constant $C$ depending on the same parameters as $C_{1}, C_{2}$ such that

$$
\left(\int_{\Omega}(y+1)|\nabla z|^{2} \mathrm{~d} x\right)^{\frac{1}{2}} \leq C \sqrt{R}
$$

leading to the desired estimate (2.4).

We show now the estimate (2.5). We use the classic $L^{\infty}$ estimates of solutions of elliptic variational equations given in Kinderlehrer and Stampacchia [9].

For any real constant $k \geq \bar{g}$, we set

$$
A(k)=\{x \in \Omega: z(x)>k\} .
$$

Since $\psi=(z-k)^{+} \in H_{0}^{1}(\Omega)$ we can take $v=\psi$ in (2.3) and obtain:

$$
\int_{\Omega}(\tilde{a}(x) y+\tilde{b}(x)) \nabla \psi \cdot \nabla \psi \mathrm{d} x=\int_{A(k)} \tilde{d}(x, y) \cdot \nabla \psi \mathrm{d} x
$$

from the above equality, $\left(H_{g}\right)\left(H_{d}\right)$, the Cauchy-Schwarz inequality and since $y \in B_{R}$ we have

$$
\begin{aligned}
\gamma \int_{\Omega}|\nabla \psi|^{2} \mathrm{~d} x & \leq \bar{d} \sqrt{R}|A(k)|^{\frac{1}{2}}\left(\int_{A(k)} y|\nabla \psi|^{2} \mathrm{~d} x\right)^{\frac{1}{2}} \\
& \leq \bar{d} \sqrt{R}|A(k)|^{\frac{1}{2}}\left(\int_{\Omega} y|\nabla z|^{2} \mathrm{~d} x\right)^{\frac{1}{2}} .
\end{aligned}
$$

Using also (2.7) we obtain

$$
\|\nabla \psi\|_{L^{2}(\Omega)}^{2} \leq \frac{C \bar{d}}{\gamma} R|A(k)|^{\frac{1}{2}} .
$$

On the other hand, for any $r$ with $4<r \leq 6$, we denote by $c_{2}(r)$ the constant satisfying the following inequality:

$$
\|v\|_{L^{r}(\Omega)} \leq c_{2}(r)\|\nabla v\|_{L^{2}(\Omega)}, \quad \forall v \in H_{0}^{1}(\Omega) .
$$

We have for any $l \geq k \geq \bar{g}$ and $r>4$ with the help of the above inequality:

$$
\begin{aligned}
(l-k)^{r}|A(l)| & =\int_{A(l)}(l-k)^{r} \mathrm{~d} x \\
& \leq \int_{A(l)}(z(x)-k)^{r} \mathrm{~d} x \\
& =\int_{A(l)}|\psi|^{r} \mathrm{~d} x \\
& \leq c_{2}(r)^{r}\|\nabla \psi\|_{L^{2}(\Omega)}^{r}
\end{aligned}
$$


From (2.8) we obtain

$$
|A(l)| \leq\left[\frac{c_{2}(r)^{2} R C \bar{d}}{\gamma}\right]^{r / 2} \frac{|A(k)|^{\frac{r}{4}}}{(l-k)^{r}}
$$

Using Lemma B1 of Kinderlehrer-Stampacchia [9], we obtain (2.5) with

$$
D=\sqrt{\frac{\left(c_{2}(r)\right) C \bar{d}}{\gamma}}|A(\bar{g})|^{\frac{r-4}{4 r}} 2^{\frac{r}{r-4}}
$$

Corollary 2.3. For $R \geq R_{1}=\left(\frac{D}{2}+\sqrt{\left.\frac{D^{2}}{4}+\bar{g}\right)}\right)^{2}$ we have $S\left(B_{R}\right) \subset B_{R}$ where $D$ is defined by (2.9).

Proof. The result is obvious from Lemma 2.2 and the fact that $S y \geq 0$ a.e. in $\Omega$.

Lemma 2.4. $T$ and $S$ are continuous from $B_{R}$ to $L^{2}(\Omega)$

Proof. Let $y \in B_{R}$ be fixed and $y_{n} \in B_{R}$ a sequence such that $y_{n} \rightarrow y$ in $L^{2}(\Omega)$ for $n \rightarrow \infty$.

Recall that

$$
0 \leq y(x) \leq R, \quad 0 \leq y_{n}(x) \leq R \quad \text { a.e. } x \in \mathbb{R}
$$

We set

$$
z=T y, z_{n}=T y_{n}
$$

The sequence $z_{n} \in g+H_{0}^{1}(\Omega)$ satisfies the variational equality

$$
\int_{\Omega}\left(\tilde{a}(x) y_{n}+\tilde{b}(x)\right) \nabla z_{n} \cdot \nabla v \mathrm{~d} x=\int_{\Omega} \tilde{d}\left(x, y_{n}\right) \cdot \nabla v \mathrm{~d} x \quad \forall v \in H_{0}^{1}(\Omega) .
$$

Setting $v=z_{n}-g$ in (2.10), we easily obtain

$$
\left\|z_{n}\right\|_{H^{1}(\Omega)} \leq C
$$

which implies the existence of $z^{*} \in g+H_{0}^{1}(\Omega)$ such that, up to a subsequence, $z_{n}$ converges to $z^{*}$ weakly in $H^{1}(\Omega)$ and strongly in $L^{2}(\Omega)$. Passing to the limit in $(2.10)$ with $v$ arbitrary in $\mathbb{D}(\Omega)$ we obtain

$$
\int_{\Omega}(\tilde{a}(x) y+\tilde{b}(x)) \nabla z^{*} \cdot \nabla v \mathrm{~d} x=\int_{\Omega} \tilde{d}(x, y) \cdot \nabla v \mathrm{~d} x \quad \forall v \in \mathbb{D}(\Omega) .
$$

By a density argument the above equality is still valid for all $v \in H_{0}^{1}(\Omega)$. Using the uniqueness of solution of (2.3) we have $z^{*}=z$ and that the entire sequence converges to $z$, which shows the continuity of $T$. The continuity of $S$ is then a direct consequence of the continuity of the operator $v \rightarrow v^{+}$from $L^{2}(\Omega)$ to $L^{2}(\Omega)$.

Lemma 2.5. $S\left(B_{R}\right)$ is relatively compact in $L^{2}(\Omega)$.

Proof. Let $z \in T\left(B_{R}\right)$. From (2.4) we have

$$
\|\nabla(z-g)\|_{L^{2}(\Omega)} \leq C
$$

From the Poincaré inequality we deduce $\|z\|_{H^{1}(\Omega)} \leq C$. Since $\left\|z^{+}\right\|_{H^{1}(\Omega)} \leq\|z\|_{H^{1}(\Omega)}$ we deduce the result.

Theorem 2.6. There exists a weak positive solution to the problem (PGEN). 
Proof. From Corollary 2.3, Lemmas 2.4, 2.5 and by using the Schauder fixed point theorem, there exists $y \in B_{R}$ a fixed point of $S$. So there exists $z \in g+H_{0}^{1}(\Omega)$ solution of $(2.3)$ with $y=z^{+}$. Taking $v=z^{-}$as a test function we have

$$
\int_{\Omega}\left(\tilde{a}(x) z^{+}+\tilde{b}(x)\right) \nabla z^{-} \cdot \nabla z^{-} \mathrm{d} x=\int_{z \geq 0} \tilde{d}\left(x, z^{+}\right) \cdot \nabla z^{-} \mathrm{d} x+\int_{z \leq 0} \tilde{d}\left(x, z^{+}\right) \cdot \nabla z^{-} \mathrm{d} x .
$$

From hypothesis $\tilde{d}(x, 0)=0$, the right hand side of the above equality is equal to 0 which implies that $z^{-}=0$, so $y=z$ is a weak positive solution of (PGEN).

\subsubsection{Uniqueness}

Theorem 2.7. We have uniqueness among all weak positive solutions to problem (PGEN). Further, suppose that $y_{i}$ is a weak solution to (PGEN) corresponding to the boundary data $g_{i}, i=1,2$. If $g_{1} \geq g_{2}$ a.e. in $\partial \Omega$, then $y_{1} \geq y_{2}$ a.e. on $\Omega$.

Proof. We set $w=y_{2}-y_{1}$ which satisfies the problem

$$
\left\{\begin{aligned}
w \in\left(g_{2}-g_{1}\right)+ & H_{0}^{1}(\Omega) \\
\int_{\Omega} \tilde{a}(x)\left(w \nabla y_{2}\right. & \left.+y_{1} \nabla w\right) \cdot \nabla v \mathrm{~d} x+\int_{\Omega} \tilde{b}(x) \nabla w \nabla v \mathrm{~d} x \\
& =\int_{\Omega}\left(\tilde{d}\left(x, y_{2}\right)-\tilde{d}\left(x, y_{1}\right)\right) \cdot \nabla v \mathrm{~d} x \quad \forall v \in H_{0}^{1}(\Omega) .
\end{aligned}\right.
$$

We remark that $w^{+} \in H_{0}^{1}(\Omega)$, so we can take as in [6,8], $v=\frac{w^{+}}{w^{+}+\epsilon}$ as a test function in $(2.11)$ with $\epsilon>0$.

Remark also that

$$
\begin{aligned}
& \nabla\left(\frac{w^{+}}{w^{+}+\epsilon}\right)=\epsilon \frac{\nabla w^{+}}{\left(w^{+}+\epsilon\right)^{2}} \\
& \nabla \log \left(1+\frac{w^{+}}{\epsilon}\right)=\frac{\nabla w^{+}}{w^{+}+\epsilon} .
\end{aligned}
$$

Using (2.12), (2.13) and the hypotheses on the data, we obtain the following four inequalities:

$$
\begin{aligned}
\int_{\Omega} \tilde{a}(x) y_{1} \nabla w \cdot \nabla v \mathrm{~d} x & =\epsilon \int_{\Omega} \tilde{a}(x) y_{1} \frac{\left|\nabla w^{+}\right|^{2}}{\left(w^{+}+\epsilon\right)^{2}} \mathrm{~d} x \geq 0 \\
\left|\int_{\Omega} \tilde{a}(x) w \nabla y_{2} \nabla v \mathrm{~d} x\right| & \leq \epsilon \int_{\Omega} \tilde{a}(x) w^{+}\left|\nabla y_{2}\right| \frac{\left|\nabla w^{+}\right|}{\left(w^{+}+\epsilon\right)^{2}} \mathrm{~d} x \\
& \leq \epsilon \bar{a} \int_{\Omega}\left|\nabla y_{2}\right|\left|\nabla \log \left(1+\frac{w^{+}}{\epsilon}\right)\right| \mathrm{d} x \\
\int_{\Omega} \tilde{b}(x) \nabla w \nabla v d x & \geq \gamma \int_{\Omega} \nabla w^{+} \nabla \frac{w^{+}}{w^{+}+\epsilon} \mathrm{d} x \\
& =\epsilon \gamma \int_{\Omega}\left|\nabla \log \left(1+\frac{w^{+}}{\epsilon}\right)\right|^{2} \mathrm{~d} x \\
\left|\int_{\Omega}\left(\tilde{d}\left(x, y_{2}\right)-\tilde{d}\left(x, y_{1}\right)\right) \cdot \nabla v \mathrm{~d} x\right| & \leq \epsilon \bar{d} \int_{\Omega}|w| \frac{\left|\nabla w^{+}\right|}{\left(w^{+}+\epsilon\right)^{2}} \mathrm{~d} x \\
& \leq \epsilon \bar{d} \sqrt{|\Omega|}|| \nabla \log \left(1+\frac{w^{+}}{\epsilon}\right) \|_{L^{2}(\Omega)} .
\end{aligned}
$$


We then deduce from $(2.11)$

$$
\gamma\left\|\nabla \log \left(1+\frac{w^{+}}{\epsilon}\right)\right\|_{L^{2}(\Omega)}^{2} \leq \bar{a}\left\|\nabla y_{2}\right\|_{L^{2}(\Omega)}\left\|\nabla \log \left(1+\frac{w^{+}}{\epsilon}\right)\right\|_{L^{2}(\Omega)}+\bar{d} \sqrt{|\Omega|}\left\|\nabla \log \left(1+\frac{w^{+}}{\epsilon}\right)\right\|_{L^{2}(\Omega)} .
$$

Since $\log \left(1+\frac{w^{+}}{\epsilon}\right) \in H_{0}^{1}(\Omega)$, from the Poincaré inequality we deduce:

$$
\int_{\Omega}\left|\log \left(1+\frac{w^{+}}{\epsilon}\right)\right|^{2} \mathrm{~d} x \leq C_{1}
$$

with $C_{1}$ independent on $\epsilon$.

We then have $w^{+}=0$ a.e. $x \in \Omega$ and the proof is complete.

\subsection{Existence and uniqueness of $(\mathrm{P})$}

We suppose that

$$
\begin{aligned}
& \left\{\begin{array}{l}
a=\left(a_{i j}\right)_{1 \leq i, j \leq n} \text { with } a_{i j}(\cdot, u) \text { measurable } \forall u \in \mathbb{R}, \\
a_{i j}(x, \cdot) \text { continuous a.e. } x \in \Omega \\
\left|a_{i j}(x, u)\right| \leq \bar{a} \text { a.e. } x \in \Omega, \forall u \in \mathbb{R} \\
\sum_{i, j} a_{i j}(x, u) \xi_{i} \xi_{j} \geq \gamma\|\xi\|^{2} \text { a.e. } x \in \Omega, \forall u \in \mathbb{R} \quad \forall \xi \in \mathbb{R}^{n}
\end{array}\right. \\
& \left\{\begin{array}{l}
b=\left(b_{i j}\right)_{1 \leq i, j \leq n} \text { with } b_{i j}(\cdot, u) \text { measurable } \forall u \in \mathbb{R}, \\
b_{i j}(x, \cdot) \text { continuous a.e. } x \in \Omega \\
\left|b_{i j}(x, u)\right| \leq \bar{b} \text { a.e. } x \in \Omega, \forall u \in \mathbb{R} \\
\sum_{i, j} b_{i j}(x, u) \xi_{i} \xi_{j} \geq \gamma\|\xi\|^{2} \text { a.e. } x \in \Omega, \forall u \in \mathbb{R} \quad \forall \xi \in \mathbb{R}^{n}
\end{array}\right. \\
& \left\{\begin{array}{l}
d=\left(d_{i}\right)_{1 \leq i \leq n} \quad \text { with } d_{i}(\cdot, u, y) \text { measurable } \forall(u, y) \in \mathbb{R} \times \mathbb{R}+ \\
d_{i}(x, \cdot, \cdot) \text { continuous a.e. } x \in \Omega d_{i}(x, u, 0)=0 \text { a.e. } x \in \Omega, \forall u \in \mathbb{R} \\
\left|d_{i}\left(x, u, y_{2}\right)-\tilde{d}_{i}\left(x, u, y_{1}\right)\right| \leq \bar{d}\left|y_{2}-y_{1}\right| \text { a.e. } x \in \Omega, \forall u \in \mathbb{R} \forall y_{1}, y_{2} \geq 0 \text { for some } \bar{d}>0 .
\end{array}\right.
\end{aligned}
$$

Corollary 2.8. Under hypotheses $\left(H_{a}\right),\left(H_{b}\right),\left(H_{d}\right)$ and $\left(H_{g}\right)$, there exists a unique weak positive solution to problem (P) for any $u \in U_{a d}$.

Proof. We apply Theorems 2.6 and 2.7 with $\tilde{a}(x)=a(x, u(x)), \tilde{b}(x)=b(x, u(x))$ and $\tilde{d}(x, y)=d(x, u(x), y)$.

\section{Some NeW RESUlts on SOME LineAR ElLiptiC PROBlems}

Theorem 3.1. Let $A \in\left(L^{\infty}(\Omega)\right)^{n^{2}}$ such that there exists $\delta>0$ satisfying

$$
\sum_{i, j=1}^{n} A_{i j}(x) \xi_{i} \xi_{j} \geq \delta\|\xi\|^{2} \quad \forall x \in \Omega \text { and } \xi \in \mathbb{R}^{n}
$$


Assume $B \in\left(L^{r}(\Omega)\right)^{n}$ with $r>n$. Then for any $f \in H^{-1}(\Omega)$, there exists a unique weak solution of the following linear elliptic problem:

$$
\left\{\begin{array}{l}
-\nabla \cdot(A(x) \nabla y)+\nabla \cdot(B(x) y)=f \\
y \in H_{0}^{1}(\Omega)
\end{array}\right.
$$

Proof. The proof of the uniqueness is the same as in Theorem 2.7. Let us show the existence.

Let $\sigma>0$ and consider the operator $D$ defined by

$$
\begin{aligned}
D: \quad H_{0}^{1} & \rightarrow H^{-1} \\
v & \mapsto g=D v=-\nabla \cdot(A(x) \nabla v)+\nabla \cdot(B(x) \cdot v)+\sigma v .
\end{aligned}
$$

Since problem (3.2) is equivalent to $D v-\sigma v=f$, we shall apply the Fredholm alternative to the operator $D$. Let

We have

$$
a(v, w)=\int_{\Omega} A(x) \nabla v \cdot \nabla w \mathrm{~d} x-\int_{\Omega} B(x) v \cdot \nabla w \mathrm{~d} x+\sigma \int_{\Omega} v w \mathrm{~d} x .
$$

$$
a(v, v) \geq \delta\|v\|_{H_{0}^{1}(\Omega)}^{2}-\int_{\Omega} B(x) v \cdot \nabla v \mathrm{~d} x+\sigma\|v\|_{L^{2}(\Omega)}^{2} .
$$

From the hypothesis we have

$$
\left|\int_{\Omega} B(x) v \cdot \nabla v \mathrm{~d} x\right| \leq\|B\|_{L^{r}(\Omega)}\|v\|_{L^{r^{\prime}(\Omega)}}\|\nabla v\|_{L^{2}(\Omega)}
$$

with

$$
\frac{1}{2}+\frac{1}{r}+\frac{1}{r^{\prime}}=1
$$

From the Sobolev embedding and interpolation inequalities, there exists $s \in] 0,1[$ such that

$$
\begin{aligned}
\|v\|_{L^{r^{\prime}}(\Omega)} & \leq C\|v\|_{H^{s}(\Omega)} \\
& \leq C\|v\|_{H^{1}(\Omega)}^{s}\|v\|_{L^{2}(\Omega)}^{1-s} .
\end{aligned}
$$

Then from the inequality $a b \leq \frac{1}{p} a^{p}+\frac{1}{q} b^{q}$ (with $p=\frac{2}{1+s}$ and $q=\frac{2}{1-s}$ ) we obtain, for any $\eta>0$

$$
\left|\int_{\Omega} B(x) v \cdot \nabla v \mathrm{~d} x\right| \leq \eta\|v\|_{H_{0}^{1}(\Omega)}^{2}+C(\eta)\|v\|_{L^{2}(\Omega)}^{2} .
$$

Then

$$
a(v, v) \geq \delta\|v\|_{H_{0}^{1}(\Omega)}^{2}-\eta\|v\|_{H_{0}^{1}(\Omega)}^{2}-C(\eta)\|v\|_{L^{2}(\Omega)}^{2}+\sigma\|v\|_{L^{2}(\Omega)}^{2} .
$$

Finally taking $\eta<\delta$, we can choose $\sigma$ sufficiently large such that $D$ is invertible. Now the Fredhom alternative gives the result.

Theorem 3.2. Let $A \in\left(L^{\infty}(\Omega)\right)^{n^{2}}$ such that there exists $\delta>0$ satisfying

$$
\sum_{i, j=1}^{n} A_{i j}(x) \xi_{i} \xi_{j} \geq \delta\|\xi\|^{2} \quad \forall x \in \Omega \text { and } \xi \in \mathbb{R}^{n}
$$


Assume $B \in\left(L^{r}(\Omega)\right)^{n}$ with $r>n$. Then for any $f \in H^{-1}(\Omega)$, there exists a unique weak solution of the following linear elliptic problem:

$$
\left\{\begin{array}{c}
-\nabla \cdot(A(x) \nabla y)+B(x) \cdot \nabla y=f \\
y \in H_{0}^{1}(\Omega) .
\end{array}\right.
$$

Proof. We can show exactly as in the above theorem that the Freedholm alternative applies here. So it suffices to prove the uniqueness which is a simple application of the following lemma.

Lemma 3.3. With the same hypotheses as in the above theorem, let $u$ in $H^{1}(\Omega)$ such that

$$
\begin{gathered}
\int_{\Omega} A(x) \nabla v \cdot \nabla w \mathrm{~d} x+\int_{\Omega} B(x) \cdot \nabla v w \mathrm{~d} x \leq 0(\text { respectively } \geq 0) \\
\forall w \in H_{0}^{1}(\Omega) \text { with } w \geq 0
\end{gathered}
$$

Then $\sup _{\Omega} v \leq \sup _{\partial \Omega} v^{+}\left(\right.$respectively $\left.\inf _{\Omega} v \geq \inf _{\partial \Omega}\left(-v^{-}\right)\right)$.

Proof. It suffices to prove the part " $\leq 0$ ". The other part is a classic consequence of the equality inf $v=$ $-\sup (-v)$. Following the proof of Theorem 8.1 of Gilbarg and Trudinger [6] we suppose that $l=\sup _{\partial \Omega} v^{+}<\sup _{\Omega} v$ and choose $k$ to satisfy $l \leq k \leq \sup _{\Omega} v$. We set $w=(v-k)^{+}$.

From (3.5) we deduce

$$
\delta\|\nabla w\|_{L^{2}(\Omega)}^{2} \leq-\int_{\Omega} B(x) \cdot \nabla w w \mathrm{~d} x .
$$

As in the proof of Theorem 3.1 we can show that for any $\eta>0$,

$$
\left|\int_{\Omega} B(x) w \cdot \nabla w \mathrm{~d} x\right| \leq C \eta\|w\|_{H_{0}^{1}(\Omega)}^{2}+C(\eta)\|w\|_{L^{2}(\Omega)}^{2} .
$$

From (3.6) and (3.7), we deduce that there exists a constant $C$ independent of $k$ such that

$$
\|w\|_{H_{0}^{1}(\Omega)} \leq C\|w\|_{L^{2}(\Omega)}^{2} .
$$

The rest of the proof is the same as in Theorem 8.1 of Gilbarg and Trudinger [6].

\section{Study of the optimal Control problem}

Let us denote by $B V(\Omega)$ the space of functions of bounded variations in $\Omega[5]$ :

$$
B V(\Omega)=\left\{v \in L^{1}(\Omega), T V(v)<+\infty\right\}
$$

with

$$
T V(v)=\sup \left\{\int_{\Omega} v \nabla \cdot \varphi \mathrm{d} x / \varphi \in\left(C_{0}^{\infty}(\Omega)\right)^{2},\|\varphi\|_{L^{\infty}(\Omega)} \leq 1\right\}
$$

where, for $\varphi=\left(\varphi_{1}, \varphi_{2}\right),\|\varphi\|_{L^{\infty}(\Omega)}=\max \left(\left\|\varphi_{1}\right\|_{L^{\infty}(\Omega)},\left\|\varphi_{2}\right\|_{L^{\infty}(\Omega)}\right)$.

$B V(\Omega)$ is a Banach space with the following norm:

$$
\|v\|_{B V(\Omega)}=\|v\|_{L^{1}(\Omega)}+T V(v) .
$$

The next properties of $B V(\Omega)$ can be found in [5]. 


\section{Proposition 4.1.}

(i) If $\left(u_{n}\right)_{n \in \mathbb{N}} \subset B V(\Omega)$ and $u_{n} \rightarrow u$ in $L^{1}(\Omega)$, then

$$
T V(u) \leq \liminf T V\left(u_{n}\right)
$$

(ii) for every $u \in B V(\Omega) \cap L^{r}(\Omega), r \in[1,+\infty)$, there exists a sequence $\left(u_{n}\right)_{n \in \mathbb{N}} \subset C^{\infty}(\bar{\Omega})$ such that $\lim _{n \rightarrow \infty} \int_{\Omega}\left|u-u_{n}\right|^{r} \mathrm{~d} x=0$ and $\lim _{n \rightarrow \infty} T V\left(u_{n}\right)=T V(u) ;$

(iii) for every bounded sequence $\left(u_{n}\right)_{n \in \mathbb{N}}^{n \rightarrow \infty} \subset B V(\Omega)$ there exists a subsequence, still denoted $u_{n}$, and a function $u \in B V(\Omega)$ such that $u_{n} \rightarrow u$ in $L^{1}(\Omega)$.

\subsection{Existence of an optimal control}

In addition to hypotheses $\left(H_{a}\right),\left(H_{b}\right),\left(H_{d}\right)$ and $\left(H_{g}\right)$ we need the following hypotheses on $F$ :

$$
F \text { is continuous from } U_{a d} \times H^{1}(\Omega) \text { to } \mathbb{R} \text {. }
$$

Proposition 4.2. If $\left(u_{n}\right)_{n \in \mathbb{N}} \subset U_{a d}$ and $u_{n} \rightarrow u$ in $L^{1}(\Omega)$, then $u \in U_{\text {ad }}$ and $u_{n} \rightarrow u$ in $L^{r}(\Omega)$, for any $r \in[1,+\infty[$.

Proof. As $u_{n}$ converges to $u$ in $L^{1}(\Omega)$ and $\alpha \leq u_{n} \leq \beta$, we have $\alpha \leq u \leq \beta$, a.e. in $\Omega$, and from Proposition 4.1 (i) we have

$$
T V(u) \leq c .
$$

Then $u \in U_{a d}$. Moreover, for any $r \in[1,+\infty[$, the following estimate:

$$
\left\|u_{n}-u\right\|_{L^{r}(\Omega)}^{r} \leq(\beta-\alpha)^{r-1}\left\|u_{n}-u\right\|_{L^{1}(\Omega)}
$$

shows that $u_{n} \rightarrow u$ in $L^{r}(\Omega)$.

Corollary 4.3. $U_{\text {ad }}$ is a compact subset of $L^{r}(\Omega)$ for any $r \in[1,+\infty[$.

Proof. Let $u_{n}$ be a sequence of $U_{a d}$, then $u_{n}$ is bounded $B V(\Omega)$. Then the corollary is a consequence of Propositions 4.2 and 4.1 (iii).

In the following, we denote by $y(u)$ the positive weak solution $y$ of $(\mathrm{P})$ corresponding to a given control $u$. We now give the main result of this section.

Theorem 4.4. The problem $(O)$ has at least one solution.

Proof. Let $\left(u_{n}\right)_{n \in \mathbb{N}} \subset U_{a d}$ a minimizing sequence and $y_{n}=y\left(u_{n}\right)$ such that

$$
F\left(u_{n}, y_{n}\right) \rightarrow F^{*}=\inf _{u \in U_{a d}} F(u, y(u)) .
$$

From Corollary 4.3, there exists a subsequence, still denoted $u_{n}$, and a function $u^{*} \in U_{a d}$ such that:

$$
u_{n} \rightarrow u^{*} \text { strongly in } L^{1}(\Omega) \text {, in } L^{2}(\Omega) \text { and } L^{\infty}(\Omega) \text { - weak-*. }
$$

Let $y_{n}=y\left(u_{n}\right) \in g+H_{0}^{1}(\Omega) \cap L^{\infty}(\Omega)$ solution of

$$
\int_{\Omega}\left(a\left(x, u_{n}\right) y_{n}+b\left(x, u_{n}\right)\right) \nabla y_{n} \cdot \nabla v \mathrm{~d} x=\int_{\Omega} d\left(x, u_{n}, y_{n}\right) \cdot \nabla v \mathrm{~d} x \quad \forall v \in H_{0}^{1}(\Omega) .
$$


From Lemma 2.2 we have

$$
\begin{aligned}
\left\|y_{n}\right\|_{H^{1}(\Omega)} & \leq C(\bar{a}, \bar{b}, \gamma, \bar{d}, \bar{g}, \Omega) \\
\left\|y_{n}\right\|_{L^{\infty}(\Omega)} & \leq C(\bar{a}, \bar{b}, \gamma, \bar{d}, \bar{g}, \Omega) .
\end{aligned}
$$

Then there exists $y \in g+H_{0}^{1}(\Omega) \cap L^{\infty}(\Omega)$ such that

$$
y_{n} \rightarrow y \text { weakly in } H^{1}(\Omega) \text { and strongly in } L^{2}(\Omega) .
$$

Since $u_{n} \rightarrow u^{*}$ in $L^{1}(\Omega)$, on a subsequence still denoted by $u_{n}$, we have

$$
u_{n}(x) \rightarrow u^{*}(x) \text { a.e. } x \in \Omega \text {. }
$$

From $\left(H_{b}\right)$ and the Lebesgue theorem we deduce

$$
b\left(x, u_{n}\right) \rightarrow b\left(x, u^{*}\right) \text { in } L^{r}(\Omega) \quad \forall r \in[1,+\infty[.
$$

In the same manner we obtain

$$
a\left(x, u_{n}\right) \rightarrow a\left(x, u^{*}\right) \text { in } L^{r}(\Omega) \quad \forall r \in[1,+\infty[.
$$

Since $y \in L^{\infty}(\Omega)$, we also obtain using $\left(H_{d}\right)$

$$
d\left(x, u_{n}, y\right) \rightarrow d\left(x, u^{*}, y\right) \text { in } L^{r}(\Omega) \quad \forall r \in[1,+\infty[.
$$

From (4.11) we easily obtain

$$
\int_{\Omega} b\left(x, u_{n}\right) \nabla y_{n} \cdot \nabla \varphi \mathrm{d} x \rightarrow \int_{\Omega} b\left(x, u^{*}\right) \nabla y \cdot \nabla \varphi \mathrm{d} x \quad \forall \varphi \in D(\Omega) .
$$

We have

with

$$
\int_{\Omega} a\left(x, u_{n}\right) y_{n} \nabla y_{n} \cdot \nabla \varphi \mathrm{d} x-\int_{\Omega} a\left(x, u^{*}\right) y \nabla y \cdot \nabla \varphi \mathrm{d} x=E_{1}^{n}+E_{2}^{n}+E_{3}^{n}
$$

$$
\begin{aligned}
& E_{1}^{n}=\int_{\Omega}\left(a\left(x, u_{n}\right)-a\left(x, u^{*}\right)\right) y_{n} \nabla y_{n} \cdot \nabla \varphi \mathrm{d} x \\
& E_{2}^{n}=\int_{\Omega} a\left(x, u^{*}\right)\left(y_{n}-y\right) \nabla y_{n} \cdot \nabla \varphi \mathrm{d} x \\
& E_{3}^{n}=\int_{\Omega} a\left(x, u^{*}\right) y \nabla\left(y_{n}-y\right) \cdot \nabla \varphi \mathrm{d} x .
\end{aligned}
$$

From (4.8) and (4.9) there exists a constant $C$ independent of $n$ such that

$$
\left\|y_{n} \nabla y_{n} \cdot \nabla \varphi\right\|_{L^{2}(\Omega)} \leq C
$$

and from (4.12), we deduce

$$
E_{1}^{n} \rightarrow 0
$$

The $L^{2}$ strong convergence of $y_{n}$ to $y$ and the estimate $\left\|a\left(x, u^{*}\right) \nabla y_{n} \cdot \nabla \varphi\right\|_{L^{2}(\Omega)} \leq C$ lead to

$$
E_{2}^{n} \rightarrow 0
$$


The weak convergence of $y_{n}$ to $y$ in $H^{1}(\Omega)$ gives $E_{3}^{n} \rightarrow 0$, so

$$
\int_{\Omega} a\left(x, h_{n}\right) y_{n} \nabla y_{n} \cdot \nabla \varphi \mathrm{d} x \rightarrow \int_{\Omega} a\left(x, h^{*}\right) y \nabla y \cdot \nabla \varphi \mathrm{d} x .
$$

We now write

$$
\begin{aligned}
\int_{\Omega}\left(d\left(x, u_{n}, y_{n}\right)-d\left(x, u^{*}, y\right)\right) \cdot \nabla \varphi \mathrm{d} x= & \int_{\Omega}\left(d\left(x, u_{n}, y_{n}\right)-d\left(x, u_{n}, y\right)\right) \cdot \nabla \varphi \mathrm{d} x \\
& +\int_{\Omega}\left(d\left(x, u_{n}, y\right)-d\left(x, u^{*}, y\right)\right) \cdot \nabla \varphi \mathrm{d} x
\end{aligned}
$$

From $\left(H_{d}\right)$, the strong convergence of $y_{n}$ to $y$ in $L^{2}(\Omega)$ and (4.13) we deduce

$$
\int_{\Omega} d\left(x, u_{n}, y_{n}\right) \cdot \nabla \varphi \mathrm{d} x \rightarrow \int_{\Omega} d\left(x, u^{*}, y\right) \cdot \nabla \varphi \mathrm{d} x
$$

Passing to the limit in (4.7) and using (4.14), (4.16) and (4.17) we obtain

$$
\int_{\Omega}\left(a\left(x, h^{*}\right) y+b\left(x, h^{*}\right)\right) \nabla y \cdot \nabla \varphi \mathrm{d} x=\int_{\Omega} d\left(x, h^{*}, y\right) \cdot \nabla \varphi \mathrm{d} x \quad \forall \varphi \in D(\Omega) .
$$

From the density of $D(\Omega)$ in $H_{0}^{1}(\Omega)$ the formulation (4.18) is still valid for all $\varphi \in H_{0}^{1}(\Omega)$; the uniqueness result (Th. 2.7) gives $y=y\left(u^{*}\right)$. From the continuity of $F$ we have

$$
F\left(u_{n}, y_{n}\right) \rightarrow F\left(u^{*}, y\right)
$$

which ends the proof.

\subsection{Optimality conditions}

We give a preliminary result which is a consequence of Theorem 4.2 p. 38 of [1].

Lemma 4.5. Let $A \in\left(L^{\infty}(\Omega)\right)^{n^{2}}$ such that there exists $\delta>0$ satisfying

$$
\sum_{i, j=1}^{n} A_{i j}(x) \xi_{i} \xi_{j} \geq \delta\|\xi\|^{2} \quad \forall x \in \Omega \text { and } \xi \in \mathbb{R}^{n} .
$$

Then there exists $r^{*}=r^{*}(\delta, \bar{A})>2$, where $\bar{A}=\max _{i, j}\left\|A_{i j}\right\|_{L^{\infty}(\Omega)}$, satisfying the following property:

For any $f \in\left(L^{r^{*}}(\Omega)\right)^{2}$ the unique solution $u$ of the problem

$$
\left\{\begin{array}{l}
\nabla \cdot(A \nabla y)=\nabla \cdot f \quad \text { in } \Omega \\
y \in H_{0}^{1}(\Omega)
\end{array}\right.
$$

belongs to $W_{0}^{1, r^{*}}(\Omega)$ and satisfy

$$
\|y\|_{W_{0}^{1, r^{*}}(\Omega)} \leq c_{1}\|f\|_{\left(L^{r^{*}}(\Omega)\right)^{2}}
$$

with $c_{1} \geq 0$ depending only on $\delta$ and $\bar{A}$.

Remark 4.6. We apply the above lemma for the problem (P) with $A=a(x, u) y+b(x, u), \delta=\gamma$ and $\bar{A}=\bar{a} R+\bar{b}$. So, there exists $s=s(\bar{a}, \bar{b}, \gamma, \bar{d}, \bar{g}, \Omega)>2$ such that, if $g \in W^{1, s}(\Omega)$ then $y \in W^{1, s}(\Omega)$. 
In this section we consider the bidimensional case $(n=2)$, since we need the Sobolev embedding $W^{1, s}(\Omega) \subset$ $L^{\infty}(\Omega)$ for any $s>2$. We also need the following supplementary assumptions on the data:

There exist $\tilde{\alpha}$ and $\tilde{\beta}$ with $0<\tilde{\alpha}<\alpha$ and $\tilde{\beta}>\beta$ such that

- $a$ and $b$ are derivable in $u$ and

$$
\left|\partial_{u} a(x, u)\right|+\left|\partial_{u} b(x, u)\right| \leq C \quad \forall x \in \Omega, \forall u \in[\tilde{\alpha}, \tilde{\beta}]
$$

- $\partial_{u} a$ and $\partial_{u} b$ are uniformly continuous on $u$ on $[\tilde{\alpha}, \tilde{\beta}]$, uniformly in $x$ in the following sense: for any $\epsilon>0$, there exists $\delta>0$ such that, if $\left|u_{1}-u_{2}\right|<\delta$ we have

$\left|\partial_{u} a\left(x, u_{1}\right)-\partial_{u} a\left(x, u_{2}\right)\right|<\epsilon\left(\operatorname{resp} .\left|\partial_{u} b\left(x, u_{1}\right)-\partial_{u} b\left(x, u_{2}\right)\right|<\epsilon\right) \quad \forall x \in \Omega, u_{1}, u_{2} \in[\tilde{\alpha}, \tilde{\beta}] ;$

(For example the product between a $L^{\infty}$ function in $x$ and a uniformly continuous function in $u$ satisfy such a condition.)

- The function $d$ is derivable in $y$ and $u$ with $\partial_{y} d$ continuous in $y$ and $\partial_{u} d$ continuous in $u$ and:

$$
\left|\partial_{y} d(x, u, y)\right|+\left|\partial_{u} d(x, u, y)\right| \leq C \quad \forall x \in \Omega, \forall u \in[\tilde{\alpha}, \tilde{\beta}], \quad \forall y \geq 0
$$

$F$ is differentiable on $U_{a d} \times H^{1}(\Omega)$;

- $g \in W^{1, s}(\Omega)$ with $s$ given in Remark 4.6.

Proposition 4.7. The mapping $u \mapsto y(u)$ is continuously differentiable from $U_{a d}$ to $W^{1, s}(\Omega)$.

Proof. To obtain the result we use the implicit function theorem. We first need to plunge $U_{a d}$ in an appropriate open set. To do that, let us consider $\tilde{c}>c$ and set

$$
\tilde{U}=\left\{u \in B V(\Omega) \cap L^{\infty}(\Omega), \tilde{\alpha}<u<\tilde{\beta} \text { and } T V(u)<\tilde{c}\right\}
$$

We now set

$$
\left.D=\operatorname{int}(\tilde{U}) \quad \text { (the interior of } \tilde{U} \text { in the topology of } B V(\Omega) \cap L^{\infty}(\Omega)\right) .
$$

It is easy to see that $U_{a d} \subset D$. We now define the mapping

$$
\begin{aligned}
G: \quad W_{0}^{1, s}(\Omega) \times D & \longrightarrow W^{-1, s}(\Omega) \\
(\bar{y}, u) & \longrightarrow-\operatorname{div}[(a(x, u)(g+\bar{y})+b(x, u)) \nabla(g+\bar{y})]+\operatorname{div}[d(x, u, g+\bar{y})]
\end{aligned}
$$

where $s$ is given by Remark 4.6 .

We first show that $G$ is differentiable in $\bar{y}$. Since $W_{0}^{1, s}(\Omega) \hookrightarrow L^{\infty}(\Omega)$ it is clear that the application

$$
\bar{y} \in W_{0}^{1, s}(\Omega) \mapsto-\operatorname{div}[(a(x, u)(g+\bar{y})+b(x, u)) \nabla(g+\bar{y})] \in W^{-1, s}(\Omega)
$$

is differentiable. Let us now show that the application

$$
\bar{y} \in W_{0}^{1, s}(\Omega) \mapsto d(x, u, g+\bar{y}) \in L^{s}(\Omega)
$$

is differentiable, which will imply the differentiability of $G$ in $\bar{y}$. We have

$$
\begin{aligned}
&\left\|d(x, u, \bar{y}+g+h)-d(x, u, \bar{y}+g)-\partial_{y} d(x, u, \bar{y}+g) h\right\|_{L^{s}(\Omega)} \\
& \leq\left\|\partial_{y} d(x, u, \bar{y}+g+\psi(x) h)-\partial_{y} d(x, u, \bar{y}+g)\right\|_{L^{s}(\Omega)} \times\|h\|_{L^{\infty}(\Omega)}
\end{aligned}
$$

with $\psi(x) \in[0,1], \forall x \in \Omega$. 
From hypotheses (4.24) and using the Lebesgue convergence theorem we obtain the differentiability. We have, for all $(\bar{y}, u) \in W_{0}^{1, s}(\Omega) \times D$ and $z \in W_{0}^{1, s}(\Omega)$ :

$$
\frac{\partial G}{\partial \bar{y}}(\bar{y}, u)(z)=-\operatorname{div}[a(x, u) \nabla(g+\bar{y}) z]+\nabla\left[\partial_{y} d(x, u, g+\bar{y}) z\right]-\operatorname{div}[(a(x, u)(g+\bar{y})+b) \nabla z] .
$$

Using hypotheses $(4.22),(4.23),(4.24)$ and by similar arguments we show that the application

$$
(\bar{y}, u) \in W_{0}^{1, s}(\Omega) \times D \mapsto \frac{\partial G}{\partial \bar{y}}(\bar{y}, u) \in \mathcal{L}\left(W_{0}^{1, s}(\Omega), W^{-1, s}(\Omega)\right)
$$

is continuous.

Now it remains to show the differentiability of $G$ in $u$ and the continuity of the differential. We show it only for the most difficult term:

$$
u \in D \mapsto b(x, u) \nabla(g+\bar{y}) \in L^{s}(\Omega)
$$

We have

$$
\left\|\left[b(x, u+h)-b(x, u)-\partial_{u} b(x, u) h\right] \nabla(g+\bar{y})\right\|_{L^{s}(\Omega)} \leq\left\|\left[\partial_{u} b(x, u+\psi(x) h)-\partial_{u} b(x, u)\right] h\right\|_{L^{\infty}(\Omega)}\|(g+\bar{y})\|_{W^{1, s}(\Omega)}
$$

with $\psi(x) \in[0,1], \forall x \in \Omega$.

Using Hypotheses (4.23) we deduce the differentiability. By similar calculations we show the differentiability of the other terms and the continuity of the differential.

So $G$ is continuously differentiable.

Finally, we have to show that for any $u \in U_{a d} \frac{\partial G}{\partial \bar{y}}(y(u)-g, u)$ is an isomorphism from $W_{0}^{1, s}(\Omega)$ to $W^{-1, s}(\Omega)$. Since $W^{-1, s}(\Omega) \subset H^{-1}(\Omega)$, we can apply Theorem 3.1 with $n=2, r=s, A=a(x, u) y(u)+b(x, u)$ (remark that $y(u) \geq 0)$ and $B=-a(x, u) \nabla y(u)+\partial_{y} d(x, u, y(u))$ which ends the proof.

Theorem 4.8. Let $u^{*}$ be a solution of problem (O). Then there exists $z \in H_{0}^{1}(\Omega)$ such that the following optimality system is satisfied:

$$
\nabla \cdot\left[\left(a\left(x, u^{*}\right) y\left(u^{*}\right)+b\left(x, u^{*}\right)\right) \nabla y\left(u^{*}\right)\right]=\nabla \cdot d\left(x, u^{*}, y\left(u^{*}\right)\right) \quad x \in \Omega, y\left(u^{*}\right) \in g+H_{0}^{1}(\Omega)
$$

$-\nabla \cdot\left[\left(a\left(x, u^{*}\right) y\left(u^{*}\right)+b\left(x, u^{*}\right)\right) \nabla z\right]+\left(a\left(x, u^{*}\right) \nabla y\left(u^{*}\right)-\partial_{y} d\left(x, u^{*}, y\left(u^{*}\right)\right)\right) \cdot \nabla z$

$$
=-\frac{\partial F}{\partial y}\left(u^{*}, y\left(u^{*}\right)\right) \quad x \in \Omega, z \in H_{0}^{1}(\Omega)
$$

$$
\frac{\mathrm{d} j}{\mathrm{~d} u}\left(u^{*}\right) \cdot\left(u-u^{*}\right) \geq 0, \quad \forall u \in U_{a d}
$$

where

$$
\begin{aligned}
\frac{\mathrm{d} j}{\mathrm{~d} u}\left(u^{*}\right) \cdot v=\int_{\Omega} v\left[\partial_{u} a\left(x, u^{*}\right)\right. & \left.y\left(u^{*}\right)+\partial_{u} b\left(x, u^{*}\right)\right] \nabla y\left(u^{*}\right) \cdot \nabla z \mathrm{~d} x \\
& -\int_{\Omega} v \partial_{u} d\left(x, u^{*}, y\left(u^{*}\right)\right) \cdot \nabla z+\partial_{u} F\left(u^{*}, y\left(u^{*}\right)\right) \cdot v \mathrm{~d} x \quad \forall v \in B V(\Omega) \cap L^{\infty}(\Omega) .
\end{aligned}
$$

Proof. Let $j: U_{a d} \rightarrow \mathbb{R}, j(u)=F(u, y(u))$. We now classically define the Lagrangian $\mathrm{\iota}$ by:

$$
\begin{aligned}
& \mathrm{L}(u, y, z)=F(u, y)+\int_{\Omega}(a(x, u) y+b(x, u)) \nabla y \cdot \nabla z \mathrm{~d} x-\int_{\Omega} d(x, u, y) \cdot \nabla z \mathrm{~d} x \\
& \forall u \in U_{a d}, y \in W^{1, s}(\Omega), z \in H_{0}^{1}(\Omega) .
\end{aligned}
$$


It is clear that $j(u)=\mathrm{E}(u, y(u), z) \forall u \in U_{a d}, z \in H_{0}^{1}(\Omega)$. From hypothesis (4.22)-(4.25), $\mathrm{E}$ is differentiable with respect to $(u, y)$. Since $u \mapsto y(u)$ is differentiable from Proposition 4.7 , we deduce the differentiability of $j$. Since $u^{*}$ is a minimum of $j$ on $U_{a d}$, we classically have (4.36). We also have

$$
\frac{\mathrm{d} j}{\mathrm{~d} u}\left(u^{*}\right) \cdot v=\partial_{u} \mathrm{E}\left(u^{*}, y\left(u^{*}\right), z\right) \cdot v+\partial_{y} \mathrm{E}\left(u^{*}, y\left(u^{*}\right), z\right) \cdot \psi
$$

with $\psi=\frac{\mathrm{d} y}{\mathrm{~d} u}\left(u^{*}\right) \cdot v$, which gives

$$
\begin{aligned}
\frac{\mathrm{d} j}{\mathrm{~d} u}\left(u^{*}\right) \cdot v & =\partial_{u} F\left(u^{*}, y\left(u^{*}\right)\right) \cdot v \\
& +\int_{\Omega} v\left[\partial_{u} a\left(x, u^{*}\right) y\left(u^{*}\right)+\partial_{u} b\left(x, u^{*}\right)\right] \nabla y\left(u^{*}\right) \cdot \nabla z \mathrm{~d} x \\
& -\int_{\Omega} v \partial_{u} d\left(x, u^{*}, y\left(u^{*}\right)\right) \cdot \nabla z+\partial_{y} F\left(u^{*}, y\left(u^{*}\right)\right) \cdot \psi \mathrm{d} x \\
& +\int_{\Omega}\left[a\left(x, u^{*}\right) y\left(u^{*}\right)+b\left(x, u^{*}\right)\right] \nabla z \cdot \nabla \psi \mathrm{d} x \\
& +\int_{\Omega}\left[a\left(x, u^{*}\right) \nabla y\left(u^{*}\right)-\partial_{y} d\left(x, u^{*}, y\left(u^{*}\right)\right)\right] \cdot \nabla z \psi \mathrm{d} x
\end{aligned}
$$

We now remark that, due to Theorem 3.2 with $n=2$ and $r=s$, the equation (4.35) has a unique solution $z \in H_{0}^{1}(\Omega)$. Since $y\left(u^{*}\right)=g+\bar{y}\left(u^{*}\right)$, with $\bar{y}\left(u^{*}\right) \in W_{0}^{1, s}(\Omega)$ (see proof of Prop. 4.7) we have $\psi \in W_{0}^{1, s}(\Omega)$. Taking $z$, the solution of the adjoint problem (4.35) with $\psi$ as a test function in its variational formulation, in (4.39), we obtain (4.37).

\section{Application to lubrication problems}

In this section we consider the lubrication problem (1.1). We suppose that $u \in U_{a d}$ as given in (1.2). It is clear that this problem is a particular case of problem $(\mathrm{P})$ and all hypotheses on data of $(\mathrm{P})$ are satisfied here. So there exists a unique positive weak solution of (1.1) denoted also by $y(u)$.

\subsection{Optimization of the head slider for maximum load}

To increase the performance of magnetic disk devices we need to find the shape of the head which gives the maximum load in order to increase the film stiffness [11]. In this case the fluctuations on the flight induced by the roughness of the disk are highly reduced. The aim of this section is then to search an optimal shape for the head under criterion "Maximal load", i.e.

$$
\text { Find } u^{*} \in U_{a d} \text { such that } j_{1}\left(u^{*}\right)=\min _{u \in U_{a d}} j_{1}(u)
$$

where

$$
j_{1}(u)=-\int_{\Omega}\left(y(u)-p_{a}\right) \mathrm{d} x .
$$

This optimisation problem is a particular case of $(\mathrm{O})$ with $F(u, y)=-\int_{\Omega}\left(y-p_{a}\right) \mathrm{d} x$. From Theorems 4.4 and 4.8 there exists an optimal solution $u^{*}$ and an adjoint $z$ satisfying

$$
\begin{gathered}
\nabla \cdot\left[\left(u^{* 3} y\left(u^{*}\right)+6 K u^{* 2}\right) \nabla y\left(u^{*}\right)\right]=\Lambda \cdot \nabla\left(u^{*} y\left(u^{*}\right)\right) \quad x \in \Omega, y\left(u^{*}\right) \in 1+H_{0}^{1}(\Omega) \\
-\nabla \cdot\left[\left(u^{* 3} y\left(u^{*}\right)+6 K u^{* 2}\right) \nabla z\right]+\left(u^{* 3} \nabla y\left(u^{*}\right)-\Lambda u^{*}\right) \cdot \nabla z=1 \quad x \in \Omega, z \in H_{0}^{1}(\Omega) \\
\frac{\mathrm{d} j_{1}}{\mathrm{~d} u}\left(u^{*}\right) \cdot\left(u-u^{*}\right) \geq 0, \forall u \in U_{a d}
\end{gathered}
$$


where

$$
\frac{\mathrm{d} j_{1}}{\mathrm{~d} u}\left(u^{*}\right) \cdot v=\int_{\Omega} v\left(3 u^{* 2} y\left(u^{*}\right)+12 K u^{*}\right) \nabla y\left(u^{*}\right) \cdot \nabla z \mathrm{~d} x-\int_{\Omega} v y\left(u^{*}\right) \Lambda \cdot \nabla z \mathrm{~d} x .
$$

\subsection{Optimization of the head slider for minimum drag forces}

Another criterion of optimality is to minimize the drag forces in the direction of the fluid motion. One of the main objectives in reducing the drag forces here is to maintain the flatness of the PFPE layer which is usually on the disk in order to make the start-stop phases easier. So the problem is

$$
\text { Find } u^{*} \in U_{a d} \text { such that } j_{2}\left(u^{*}\right)=\min _{u \in U_{a d}} j_{2}(u)
$$

where

$$
j_{2}(u)=\left(\int_{\Omega}\left(\frac{1}{u}-\frac{3 u}{\Lambda_{1}} \frac{\partial y(u)}{\partial x_{1}}\right) \mathrm{d} x\right)^{2}
$$

This is also a particular case of problem $(\mathrm{O})$ for which we can apply the general results.

For this problem the optimal solution is given by:

$$
\begin{gathered}
\nabla \cdot\left[\left(u^{* 3} y\left(u^{*}\right)+6 K u^{* 2}\right) \nabla y\left(u^{*}\right)\right]=\Lambda \cdot \nabla\left(u^{*} y\left(u^{*}\right)\right) \quad x \in \Omega, y\left(u^{*}\right) \in 1+H_{0}^{1}(\Omega) \\
-\nabla \cdot\left[\left(u^{* 3} y\left(u^{*}\right)+6 K u^{* 2}\right) \nabla z\right]+\left(u^{* 3} \nabla y\left(u^{*}\right)-\Lambda u^{*}\right) \cdot \nabla z=-\frac{6}{\Lambda_{1}} \frac{\partial u}{\partial x_{1}} \int_{\Omega}\left(\frac{1}{u}-\frac{3 u}{\Lambda_{1}} \frac{\partial y(u)}{\partial x_{1}}\right) \mathrm{d} x \quad z \in H_{0}^{1}(\Omega) \\
\frac{\mathrm{d} j_{2}}{\mathrm{~d} u}\left(u^{*}\right) \cdot\left(u-u^{*}\right) \geq 0, \quad \forall u \in U_{a d}
\end{gathered}
$$

where

$$
\begin{aligned}
\frac{\mathrm{d} j_{2}}{\mathrm{~d} u}\left(u^{*}\right) \cdot v=\int_{\Omega} v\left(3 u^{* 2} y\left(u^{*}\right)+12 K u^{*}\right) \nabla y\left(u^{*}\right) & \cdot \nabla z \mathrm{~d} x-\int_{\Omega} v y\left(u^{*}\right) \Lambda \cdot \nabla z \mathrm{~d} x \\
& +\int_{\Omega}\left(-\frac{1}{u^{2}}-\frac{3}{\Lambda_{1}} \frac{\partial y(u)}{\partial x_{1}}\right) v \mathrm{~d} x \int_{\Omega}\left(\frac{1}{u}-\frac{3 u}{\Lambda_{1}} \frac{\partial y(u)}{\partial x_{1}}\right) \mathrm{d} x .
\end{aligned}
$$

Remark 5.1. In practical situations we look for a compromise of both criterion: maximizing the load and minimizing the drag forces. We can take for example:

$$
\text { Find } u^{*} \in U_{a d} \text { such that } j_{3}\left(u^{*}\right)=\min _{u \in U_{a d}} j_{3}(u)
$$

where

$$
j_{3}(u)=\mu j_{1}(u)+(1-\mu) j_{2}(u) \quad \mu \in[0,1] .
$$

The choice of $\mu$ depends on the importance of criterion $j_{1}$ and $j_{2}$ (a smaller $\mu$ corresponds to a smaller importance of the load). 


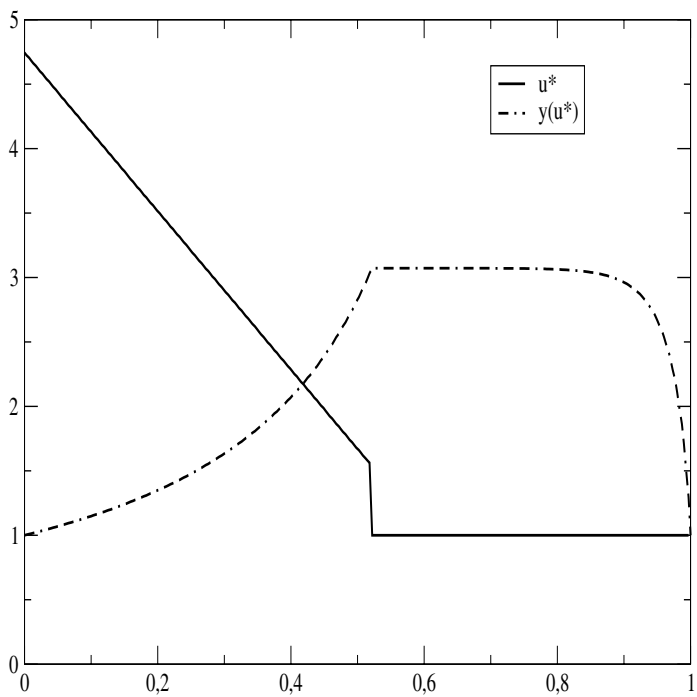

FiguRE 1. Optimal control and corresponding pressure profile. $\Lambda=100, K=1, \beta=10, c=1000$.

\section{NUMERICAL RESULTS}

In this section we find numerically an optimal head shape which maximizes the load (Sect. 5.1) in the one dimensional case. So the state equation becomes

$$
\begin{aligned}
\frac{\mathrm{d}}{\mathrm{d} x}\left(\left(u^{3} y+6 K u^{2}\right) \frac{\mathrm{d} y}{\mathrm{~d} x}\right) & =\Lambda \frac{\mathrm{d}}{\mathrm{d} x}(u y) \\
y(0) & =y(1)=1 .
\end{aligned}
$$

We consider a regular subdivision of $[0,1]\left(x_{i}=i h, i=0, \ldots, n+1: h=\frac{1}{n+1}\right)$.

An approximation of the function $u \in U_{a d}$ is of the form:

$$
u^{n}(x)=\sum_{i=1}^{n+1} u_{i} \chi_{i}(x)
$$

where $\chi_{i}$ is the characteristic function of $I_{i}=\left[x_{i-1}, x_{i}\right]$.

Remark that

$$
T V\left(u^{n}\right)=\sum_{i=1}^{n}\left|u_{i+1}-u_{i}\right|
$$

We solve problem (6.1) using the $P 1$ finite elements and fixed point iterations. We then solve the corresponding adjoint problem (4.35) and we evaluate $j_{1}$ and its gradient. We then use the procedure DONLP2 from Netlib library to minimize $j_{1}$.

We observe in Figures 1 and 2 that increasing $\Lambda$ leads to an increasing of the entrance point $\left(u^{*}(0)\right)$. Actually the number $\Lambda$ is very high due to the very thin film thickness. In order to avoid a large entrance point we take a smaller $\beta$ for which we obtain a saturated optimal shape (see Fig. 3).

The above results are obtained with a high value of the bound on the total variation $(c=1000)$. The same result is obtained for greater value of $c$ so that the solution is not depending of the value. For smaller value of $c$ (see Fig. 4), the constraint due to the total variation is saturated and the optimal solution is different. 


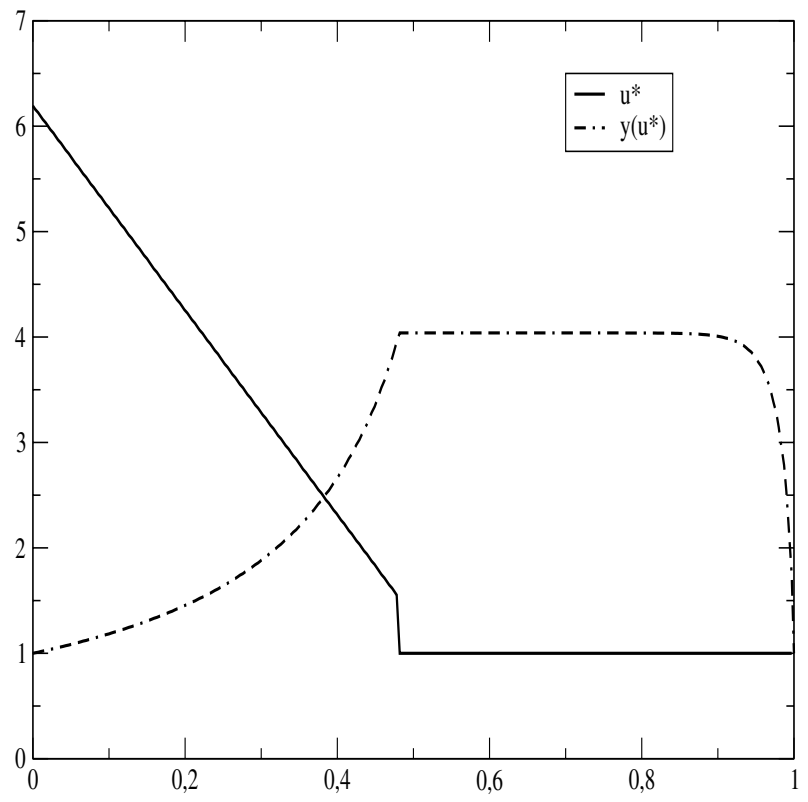

Figure 2. Optimal control and corresponding pressure profile. $\Lambda=$ $200, K=1, \beta=10, c=1000$.

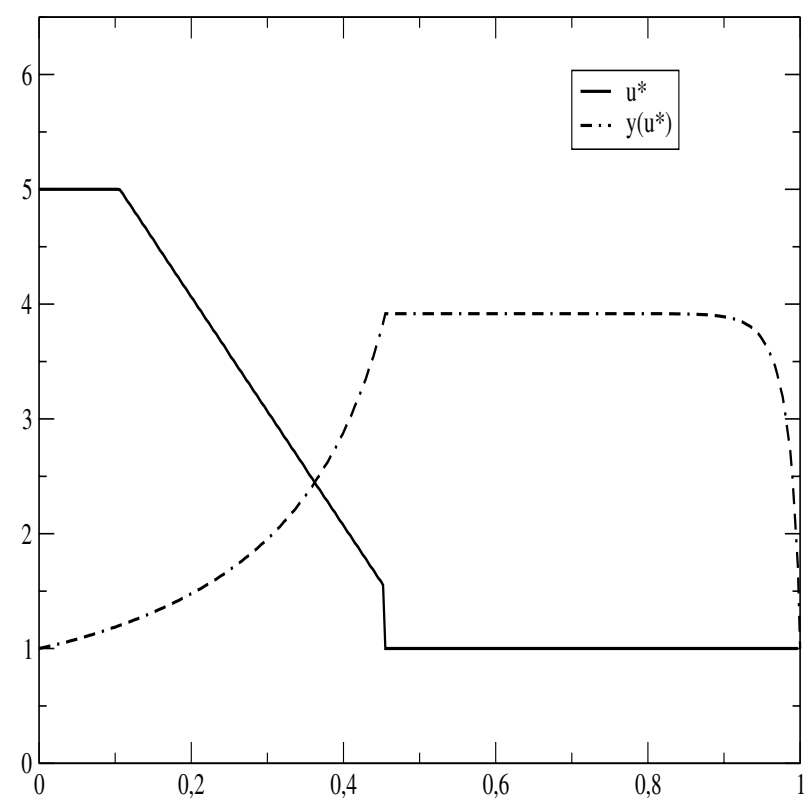

Figure 3. Optimal control and corresponding pressure profile. $\Lambda=$ $200, K=1, \beta=5, c=1000$.

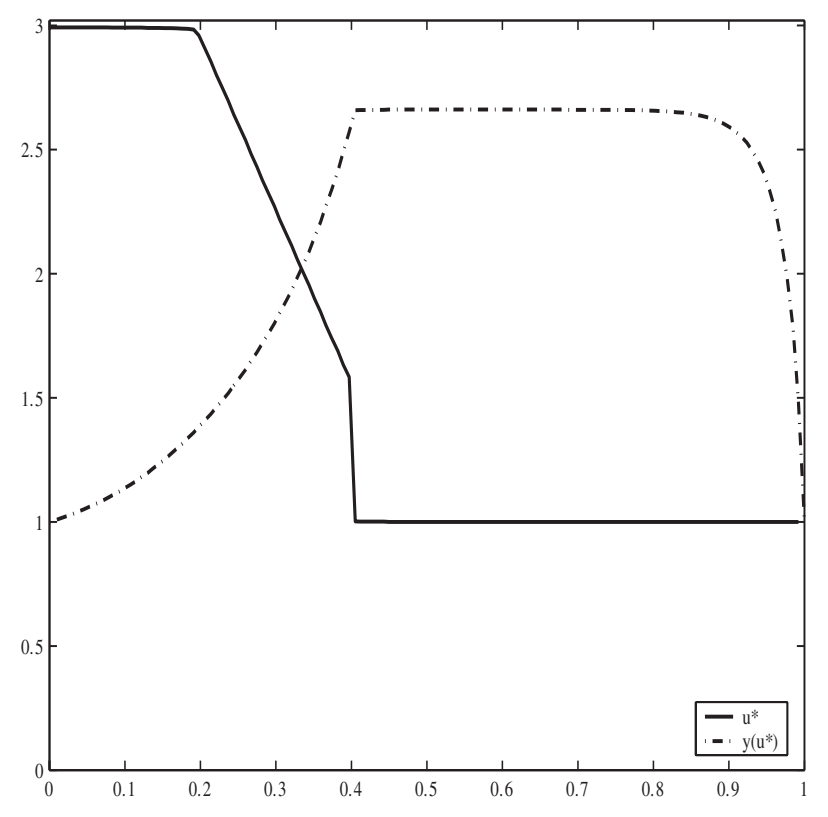

Figure 4. Optimal control and corresponding pressure profile. $\Lambda=$ $100, K=1, \beta=10, c=2$.

An interesting and open problem could be to prove the existence of an optimal control by considering the new admissible control set: $U_{a d}=\left\{u \in L^{\infty}(\Omega), \alpha \leq u \leq \beta\right\}$. 


\section{REFERENCES}

[1] A. Bensoussan, J.L. Lions and G. Papanicolaou, Asymptotic analysis for periodic structure. North-Holland (1978).

[2] B. Burgdorfer, The influence of the molecular mean free path on the performance of hydrodynamic gas lubricated bearings. ASME J. basic Engineer. 81 (1959) 99-100.

[3] M. Chipot and M. Luskin, Existence and uniqueness of solutions to the compressible Reynolds lubrication equation. SIAM J. Math. Anal. 17 (1986) 1390-1399.

[4] J.I. Diaz and J.I. Tello, On a problem lacking a classical solution in lubrication theory, in Actas del XV-CEDYA, Vigo II (1997) 429-434.

[5] L.C. Evans and R.F. Gariepy, Measure theory and fine properties of functions. Stud. Adv. Math. CRC Press (1992).

[6] D. Gilbarg and N.S. Trudinger, Elliptic partial differential equations of second order. Springer-Verlag, Berlin, second edition (1983).

[7] B.S. Grigor'ev, S.V. Lupulyak and Yu.K. Shinder, Solvability of the reynolds equation of gas lubrication. J. Math. Sci. 106 (2001) 2925-2928.

[8] M. Jai, Existence and uniqueness of solutions of the parabolic nonlinear compressible Reynolds lubrication equation. Nonlinear Anal. 43 (2001) 655-682.

[9] D. Kinderlehrer and G. Stampacchia, An introduction to variational inequalities and their applications. Academic Press Inc. [Harcourt Brace Jovanovich Publishers], New York (1980).

[10] L. Rayleigh, Notes on the Theory of Lubrication. Phylosophical Magazine 35 (1918) 1-12.

[11] M.P. Robert, Optimization of self-acting gas bearings for maximum static siffness. ASME J. Appl. Mech. 57 (1990) $758-761$.

[12] S.M. Rodhe and G.T. McAllister, On the optimization of fluid film bearings. Proc. Roy. Soc. London A 351 (1976) $481-497$.

[13] J. I. Tello, Regularity of solutions to a lubrication problem with discontinuous separation data. Nonlinear Anal. 53 (2003) $1167-1177$. 